Assessment of Treatment Efficacy Immediately After Combined Therapy of Ultrasound-Guided Radiofrequency Ablation and Conventional Transcatheter Arterial Chemoembolization for Hepatocellular Carcinoma: Comparison Between Multidetector-Row

\title{
CT and MRI
}

\author{
Sung Mo Kim ${ }^{1}$, Sang Soo Shin $\mathbb{D}^{1,2,{ }^{*}, \text { Byung Chan } \text { Lee }^{1} \text {, Jin Woong Kim }}{ }^{3}$, Suk Hee Heo ${ }^{1}$, Chung Hwan \\ Jun $^{4}$ and Yong Yeon Jeong ${ }^{1}$ \\ ${ }^{1}$ Department of Radiology, Chonnam National University Hospital and Medical School, Gwangju, South Korea \\ ${ }^{2}$ Center for Aging and Geriatrics, Chonnam National University Hospital and Medical School, Gwangju, South Korea \\ ${ }^{3}$ Department of Radiology, Chosun University Hospital, Gwangju, South Korea \\ ${ }^{4}$ Department of Gastroenterology, Chonnam National University Hospital and Medical School, Gwangju, South Korea \\ Corresponding author: Department of Radiology, Chonnam National University Hospital, Chonnam National University Medical School, 42 Jebong-ro, Dong-gu, Gwangju, \\ South Korea. Tel: +82-622205882, Fax: +82-622264380, Email: kjradsss@gmail.com \\ Received 2018 June 17; Revised 2018 December 10; Accepted 2019 February 10.
}

\begin{abstract}
Background: In the case of combined therapy, potential accumulation of iodized oil (lipiodol) in the liver parenchyma around the index tumor may directly interfere with the imaging evaluation of the treatment efficacy immediately after the procedure. We postulated that magnetic resonance imaging (MRI) has the potential to precisely evaluate the ablation zone immediately after radiofrequency ablation (RFA) combined with conventional transcatheter arterial chemoembolization (TACE) for hepatocellular carcinoma (HCC) because the effect of lipiodol retention on MRI signal intensity is minimal.

Objectives: To prospectively compare multidetector-row CT (MDCT) and MRI for evaluation of the ablative margin (AM) and index tumor immediately after combined treatment with conventional TACE and ultrasound-guided RFA for HCC.

Patients and Methods: This study included 33 consecutive patients with 45 HCCs in whom both contrast-enhanced MDCT and MRI were performed immediately after RFA combined with TACE. Two radiologists independently reviewed the images in terms of the ease of visually discriminating between the AM and index tumor (positive versus negative target sign) and the AM status within the RFA zone. The AM status was divided into AM-plus (completely surrounding tumor), AM-zero (partly discontinuous, without bulging tumor portion), and AM-minus (partly discontinuous, with bulging tumor portion). The McNemar test and kappa statistics were used to compare the CT and MRI data for the incidence of a target sign. The clinical and imaging features were analyzed for the correlation with the local tumor progression using univariate and multivariate analysis.

Results: The AM and index tumor were visually discriminated within the ablation zone in 36 (80\%) and 40 (88.9\%) of 45 ablation zones on MDCT and MRI, respectively $(\mathrm{P}=0.387$; slight agreement, $\mathrm{k}=0.0)$. On the basis of the results of MDCT and MRI, AM status was classified as AM plus ( $\mathrm{n}=27$ and 31, respectively) and AM zero $(\mathrm{n}=9$ each). Local tumor progression occurred significantly less in cases with AM-plus on MDCT $(\mathrm{P}=0.015)$ and MRI $(\mathrm{P}=0.023)$ during the follow-up period.

Conclusion: MDCT and MRI had equivalent ability to differentiate between the AM and index tumor within the ablation zones immediately after RFA combined with TACE.
\end{abstract}

Keywords: Hepatocellular Carcinoma, Radiofrequency Ablation, Transcatheter Arterial Chemoembolization, Combination Therapy, Ablative Margin, MDCT, MRI

\section{Background}

Since its introduction in clinical practice in the mid1990s, radiofrequency ablation (RFA) has been effectively used in the management of patients with hepatocellular carcinoma (HCC) and liver metastasis (1-6). However, insufficient ablation may eventually result in increased local tumor progression (LTP) $(7,8)$. Among many predictors for LTP, the amount of the ablative margin (AM) has been re- 
ported to be the most significant factor associated with LTP (8-12). To ensure success of the procedure, it is generally required that in addition to ablating the tumor, the normal hepatic parenchyma surrounding the index tumor is included in the ablation zone to a depth of at least $3-5 \mathrm{~mm}$ $(9,10)$. Since the LTP tends to occur increasingly in proportion to the tumor diameter, the ultimate goal of the procedure should be to obtain a sufficient size of the ablated zone, especially in HCCs $>2-3 \mathrm{~cm}$ (13-15). Combined treatment with conventional transcatheter arterial chemoembolization (TACE) and RFA has been reported to be a useful method to expand the RFA zone $(13,16)$.

Among various imaging modalities, contrastenhanced CT is routinely used to determine technical success of combined therapy, which involves side-by-side review of post-procedural CT and pre-procedural initial CT, as with RFA alone $(9,17-19)$. However, in the case of combined therapy, potential accumulation of iodized oil (lipiodol) in the liver parenchyma around the index tumor may directly interfere with imaging evaluation of the treatment efficacy immediately after the procedure (20). During the last decade, several new strategies using MRI to assess treatment response to RFA alone or combined TACE and RFA therapy have been proposed (17, 21-25). magnetic resonance imaging (MRI) has the potential to precisely evaluate the ablation zone immediately after RFA combined with TACE because the effect of lipiodol retention on MRI signal intensity (SI) is minimal (26).

However, few studies have prospectively compared the ability of contrast-enhanced multidetector-row CT (MDCT) with MRI immediately after the procedure to visually differentiate between the AM and index tumor within the RFA zone only in patients who have undergone combined therapy with conventional TACE and RFA.

\section{Objectives}

The purpose of this study was to prospectively compare MDCT and MRI for evaluation of the AM and index tumor immediately after combined therapy of conventional TACE and ultrasound-guided RFA in patients with HCC.

\section{Patients and Methods}

\subsection{Study Design and Patient Population}

This study was a prospective investigation of MDCT and MRI findings of patients who underwent combined therapy of conventional TACE and RFA for HCC at our clinic over the course of 15 months. The study protocol was approved by our institutional review board, and all patients gave written informed consent.
The inclusion criteria were: (1) presence of $\leq 3$ HCCs with largest dimension of $2-5 \mathrm{~cm}$ [13], (2) Child-Pugh class A or B, (3) no tumoral invasion of the portal and hepatic veins, (4) no evidence of metastasis outside the liver, (5) prothrombin time ratio $\geq 50 \%$, (6) platelet count $\geq$ 50000 cells $/ \mathrm{mm}^{3}$, and (7) HCCs accessible with the guidance of percutaneous ultrasonography $(9,13)$. During the 15-month study period, 59 consecutive patients underwent combined treatment with TACE and percutaneous ultrasound-guided RFA at our clinic. Of the 59 patients, 26 were not included in this study because of failure to provide written informed consent $(n=22)$, inability to undergo MRI due to poor respiratory cooperation $(n=3)$, or claustrophobia $(\mathrm{n}=1)$ (Figure 1). Therefore, 33 consecutive patients (27 men and 6 women; age range, 45 - 80 years; mean age, $62.6 \pm 9.3$ years) with 45 HCCs in whom both contrast-enhanced MDCT and MRI were performed immediately after RFA combined with TACE were finally enrolled (Table 1). With respect to the number of HCCs, 21 patients had a single HCC lesion, whereas 12 patients had two HCCs. The mean size of the HCCs was $2.88 \pm 0.64 \mathrm{~cm}$. Diagnosis of the 45 HCCs was confirmed by percutaneous biopsy $(\mathrm{n}=7)$ or typical imaging findings (arterial phase hyperenhancement accompanied by delayed washout) $(\mathrm{n}=38)$ (27-30).

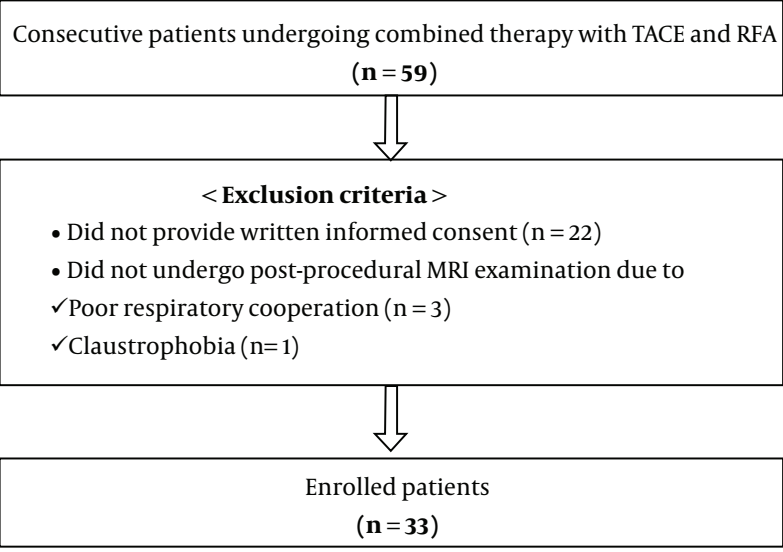

Figure 1. Flow diagram showing selection process for patients enrolled in this study (RFA, radiofrequency ablation; TACE, transcatheter arterial chemoembolization)

\subsection{Transcatheter Arterial Chemoembolization}

One interventional radiologist performed TACE using the digital subtraction method (Advantx LCA/LP+; GE Healthcare or Allura Xper FD20; Philips Medical Systems). After localizing the hepatic arterial branches that supply blood flow to the HCCs through common hepatic 


\begin{tabular}{|c|c|}
\hline Characteristics & No. of cases \\
\hline \multicolumn{2}{|c|}{ Patients $(n=33)$} \\
\hline \multicolumn{2}{|l|}{ Age, y } \\
\hline Mean $\pm S D$ & $63.3 \pm 9.5$ \\
\hline Range & $45-80$ \\
\hline \multicolumn{2}{|l|}{ Gender } \\
\hline Male & 26 \\
\hline Female & 7 \\
\hline \multicolumn{2}{|l|}{ Etiology of cirrhosis } \\
\hline Hepatitis B virus & 23 \\
\hline Hepatitis C virus & 6 \\
\hline Alcohol & 4 \\
\hline \multicolumn{2}{|l|}{ Child-pugh class } \\
\hline Class A & 33 \\
\hline \multicolumn{2}{|c|}{ Number of HCC per patient } \\
\hline Single & 21 \\
\hline Two & 12 \\
\hline & \\
\hline
\end{tabular}

Diameter, $\mathrm{cm}$

\begin{tabular}{|c|c|}
\hline Mean \pm SD & $2.88 \pm 0.64$ \\
\hline Range & $2-5$ \\
\hline \multicolumn{2}{|l|}{ Confirmation method } \\
\hline Histology & 7 \\
\hline $\mathrm{CT}$ and MR imaging findings & 38 \\
\hline \multicolumn{2}{|l|}{ Capsule } \\
\hline Present/absent & $36 / 9$ \\
\hline \multicolumn{2}{|l|}{ Location } \\
\hline Segment 2 & 4 \\
\hline Segment 3 & 3 \\
\hline Segment 4 & 4 \\
\hline Segment 5 & 6 \\
\hline Segment 6 & 9 \\
\hline Segment 7 & 5 \\
\hline Segment 8 & 14 \\
\hline \multicolumn{2}{|l|}{ Signal intensity on MRI } \\
\hline In-phase T1WI (low/iso/high) & $35 / 6 / 4$ \\
\hline Opposed-phase T1WI (low/iso/high) & $31 / 9 / 5$ \\
\hline T2WI (low/iso/high) & $2 / 5 / 38$ \\
\hline
\end{tabular}

Abbreviations: HCC, hepatocellular carcinoma; MRI, magnetic resonance imaging; SD, standard deviation; T1WI, T1 weighted image; T2WI, T2 weighted image artery angiography, TACE was conducted after advancing the catheter tip as close to the tumor as possible. For chemoembolization, emulsions of doxorubicin hydrochloride (10 mg) (Adriamycin; Ildong Co. Ltd., Seoul, Korea) and iodized oil (Lipiodol; Guerbet Laboratories, Anulnay-sous-Bois, France) were slowly administered under fluoroscopic guidance until iodized oil appeared in the portal vein branches. The amount of iodized oil was adjusted per size of the index tumor, with a maximum dose of $10 \mathrm{cc}$. At the end of the procedure, the feeding artery was embolized using Gelfoam powder (Cutanplast; Mascia Brunelli, Milan, Italy) to enhance the effect of chemoembolization.

\subsection{Radiofrequency Ablation}

RFA was performed 1 day after TACE in all patients. All RFA procedures were conducted under percutaneous ultrasound guidance through a 5 - $1 \mathrm{MHz}$ convex probe. During the procedure, the patients' hemodynamic status was continuously monitored. Depending on the tumor location, the intercostal or subcostal approach was used for inserting the electrodes under sonographic guidance. The most appropriate approach was chosen such that large vessels near the index tumor were avoided. The artificial ascites technique was performed in 26 (78.8\%) of the 33 patients (31). The procedure was performed using a monopolar 200-W radiofrequency generator (VIVA RF Generator; STARmed Co. Ltd. Koyang, Gyeonggi Province, Korea) with the consecutive activation mode and internally cooled electrodes (Well point; STARmed Co. Ltd., Koyang, Gyeonggi Province, Korea). RFA was considered complete when the location of the index tumor was completely covered by a transient hyperechoic cloud on the ultrasound images.

\subsection{MDCT and MRI}

After completing RFA, MDCT and MRI were performed within 3 and 7 hours, respectively. CT studies were performed using one of two 64-section CT machines (Sensation Cardiac 64, Siemens Medical System or Discovery CT750 HD, GE Healthcare). We randomly assigned one of two CT machines to patients, because the precision and imaging power of two CT machines in the post-procedural evaluation of the liver were considered similar on the basis of preliminary imaging study as well as daily clinical practice. After obtaining unenhanced CT from the hepatic dome to the right hepatic angle, triple-phase (including the arterial, portal venous, and delayed phases) contrast-enhanced CT was performed after intravenous injection of iodinated contrast material, $100-150 \mathrm{~mL}$ at 3.5 $\mathrm{mL} / \mathrm{sec}$ through the antecubital vein. Arterial-phase scanning was performed $18 \mathrm{sec}$ after the trigger threshold (100 
Hounsfield unit [HU] on the abdominal aorta) was reached. The scan delay for the portal venous and delayed-phase scanning was set at 70-80 and $150-180 \mathrm{sec}$, respectively, after injection of the contrast media.

Scanning parameters for the contrast-enhanced imaging were as follows: beam pitch: $0.8 / 0.984$; nominal section thickness: $3 / 3.75 \mathrm{~mm}$; table speed: $30.72 / 40 \mathrm{~mm}$ per rotation; gantry rotation time: $0.5 / 0.5 \mathrm{sec}$; reconstruction interval: $3 / 3.75 \mathrm{~mm}$; tube current: $120 \sim 350 \mathrm{~mA}$ (depending on the body habitus of the patient) and tube voltage: $120 / 120 \mathrm{kV}$. Coronal reformatted CT images with 2-mm section-thickness and 2-mm interval were generated for all patients using dedicated software (Advantage Workstation 4.2, GE Healthcare; Navigator, Siemens Medical System).

MRI was performed in all patients using a 3-T MR machine (Magnetom TrioTim; Siemens Medical System, Forchheim, Germany). After obtaining pre-contrast T1- and T2weighted MR images (axial, coronal, and sagittal planes), patients subsequently underwent contrast-enhanced MRI after intravenous injection of a standard volume (0.1 $\mathrm{mmol} / \mathrm{kg}$ ) of nonspecific extracellular contrast material (gadoterate meglumine, Dotarem, Guerbet) at flow-rate 1.5 $\mathrm{mL} / \mathrm{sec}$. After injection of contrast media, scan delay for the arterial, portal, and delayed phases was set at 30, 60, and 100 seconds, respectively. Coronal and sagittal images were additionally obtained at 140 and 180 seconds, respectively. Table 2 shows the imaging parameters of each MRI scan protocol.

\subsection{Treatment Course and Follow-Up}

In cases with immediate post-procedural images showing tumor enhancement and washout around the RFA zone, additional ablation was done to achieve complete ablation of the remnant tumor. Complete coverage of the index HCC by the ablation zone with absence of residual tumor around the RFA zone was regarded as technical success; and absence of nodular arterial enhancement around the RFA zone at 1-month follow-up CT was considered as confirmed technical efficacy (32). In cases with confirmed radiological technical efficacy, imaging surveillance was performed using CT or MRI every 3 months. Patients in whom LTP (newly developed tumor enhancement and washout in or along the RFA zone) and/or new HCCs in the liver were detected were treated with RFA, TACE, or systemic chemotherapy based on the patients' clinical scenario.

\subsection{Image Analysis}

Two radiologists independently reviewed both $\mathrm{CT}$ and MR images in two separate sessions, in order. The interval between two reading sessions was set at 6 weeks to

\begin{tabular}{|ll}
\hline Table 2. Imaging Parameters of Various MR Imaging Protocols \\
\hline Imaging parameters & 3T (Magnetom TrioTim) \\
\hline T1-weighted imaging (VIBE) & \\
\hline TR/TE, ms (in-phase/opposed phase) & $3.8 / 2.2 / 1.3$ \\
\hline Section thickness, mm & 3 \\
\hline Flip angle, deg & 9 \\
\hline FOV & $360 \times 360$ \\
\hline Matrix number & $320 \times 240$ \\
\hline T2-weighted imaging(HASTE) & \\
\hline TR/TE, ms & $2000 / 169$ \\
\hline Section thickness, mm & 5 \\
\hline Interslice gap, mm & 0.5 \\
\hline Flip angle, deg & 150 \\
\hline FOV & $360 \times 360$ \\
\hline Matrix number & $320 \times 256$ \\
\hline Contrast-enhanced imaging (VIBE) & $3.4 / 1.23$ \\
\hline TR/TE, ms & 3 \\
\hline Section thickness, mm & 13 \\
\hline Flip angle, deg & $360 \times 300$ \\
\hline FOV & $320 \times 195$ \\
\hline Matrix number & \\
\hline
\end{tabular}

Abbreviations: deg, degree $\left({ }^{\circ}\right)$; HASTE, half-fourier acquisition single-shot turbo-spin echo; FOV, field of view; TR, repetition time; TE, echo time; VIBE, volumetric interpolated breath-hold examination

minimize recall bias. Inter-observer disagreement with regard to image interpretation was resolved on the basis of a third radiologist's decision. When necessary, the reviewers changed window width and level on the picture archiving and communication system workstation (Marotech 5.4, Seoul, Korea) to determine whether it was possible to detect and localize the index tumor within the ablation zone. Ease of visual discrimination between the AM and index tumor within the RFA zone was categorized as easy (positive target sign) if the index tumor was clearly identified with similar size and shape to those at preprocedural imaging or as difficult (negative target sign) if these criteria were not fulfilled. In cases with positive target sign, the ablation zones were further evaluated with respect to the AM status, which consisted of AM-plus (completely surrounding tumor), AM-zero (partly discontinuous, without bulging tumor portion), and AM-minus (partly discontinuous, with bulging tumor portion) (23).

In MDCT to determine the presence of target sign and AM status, among multiphasic images, portal venous phase images were primarily reviewed. However, in cases with AM status of either AM-zero or AM-minus, arterial and 
delayed phase images were additionally used to assess the presence or absence of arterial enhancing residual tumor portion accompanied by delayed washout around the ablated zones, respectively. In MR imaging, RFA zones were reviewed primarily using pre-contrast T1-weighted (T1W) chemical shift imaging as well as T2-weighted imaging (T2WI). In cases with AM status other than AM-plus on precontrast images, post-contrast MR images were evaluated together with pre-contrast images. In addition, the incidence of the target sign in the MR sequences was correlated with the SI of index tumor at pre-RFA MRI.

\subsection{Statistical Analysis}

All data were analyzed using Medcalc Version 18.9.1 computer software (Mariakerke, Belgium). Cumulative LTP was calculated using the Kaplan-Meier method. Univariate and multivariate Cox proportional hazards regression were used to evaluate the independent effects of various clinical and imaging features on the LTP. The McNemar test was used to compare the incidence of the target sign between CT and MRI data. Cochran's Q test was used to determine differences in the presence of positive target sign among the MRI sequences. The log-rank test was used to compare the rates of LTP according to the AM status. The degree of agreement between MDCT and MRI for the incidence of the target sign and classifying the AM status was expressed using kappa coefficients. Statistical significances were considered present when p values were < 0.05 .

\section{Results}

No additional RFA was required in any case on the basis of the findings from immediate post-procedural images. At 1-month follow-up CT, technical efficacy rate was 100\% (45 of 45 lesions). During the follow-up period (range, 12.8 to 32.5 months; mean \pm standard deviation, 22 months \pm 4.7), LTP occurred in five (11.1\%) of the 45 RFA zones. Cumulative LTP rates were $0 \%$ and $9.1 \%$ at 1 and 2 years, respectively.

Regarding the effects of various clinical and imaging features, including the etiology of cirrhosis, Child-Pugh class, size and location of HCCs, presence or absence of capsule, and AM status on MDCT and MRI on the occurrence of LTP, multivariate analysis revealed that the AM status on both MDCT $(\mathrm{P}=0.015)$ and MRI $(\mathrm{P}=0.023)$ were statistically significant factors.

Overall, three cases were referred to third radiologist for final diagnosis. One case was interpreted as having positive and negative target sign on CT images by both reviewers, respectively. This case was finally determined as having negative target sign by third radiologist. However, on MR images, both reviewers equally classified this case into a case with positive target sign and AM-plus status. One case was classified into AM-plus and AM-zero on CT images by both reviewers, respectively. This case was finally categorized as AM-zero by third radiologist. On MR images, this case was equally interpreted as a case showing AM-plus status by both reviewers. One case was classified into AM-plus and AM-zero on MR images by both reviewers, respectively. This case was finally categorized as AM-zero by third radiologist. Both reviewers equally classified this case as AM-zero on CT images.

\subsection{Comparison Between MDCT and MRI Regarding Presence of Target Sign}

MDCT and MRI revealed positive target sign in $36(80 \%)$ and $40(88.9 \%)$ of the 45 RFA zones and negative target sign in nine $(20 \%)$ and five $(11.1 \%)$ cases, respectively $(\mathrm{P}=0.387)$ (slight agreement; $\mathrm{k}=0.0,95 \%$ confidence interval $=-0.275$ $\sim 0.275$ ) (Figures 2-4). In the 36 RFA zones with a positive target sign on CT images, the index tumor appeared as a compact $(\mathrm{n}=28)$ or defective $(\mathrm{n}=8)$ lipiodol-retaining lesion surrounded by the peripheral low-attenuating portion of the RFA zone (Figure 2). The size and shape of all lipiodol-retaining lesions were matched with the HCCs visualized on pre-treatment CT images based on the longest and shortest dimension of the lesions, and visual perception in shape of the lesions. In nine RFA zones, it was difficult to visually separate the index tumor from the AM due to amorphic and dense accumulation of hyperattenuating iodized oil around the index tumor $(n=7)$ or insufficient lipiodol retention within the tumor $(n=2)$.

Among 40 cases with positive target sign on MR images, the incidence of positive target sign was significantly higher through in-phase $(\mathrm{n}=38,84.4 \%)$ and opposed-phase T1WI $(\mathrm{n}=38,84.4 \%)$ than T2WI $(\mathrm{n}=10,22.2 \%)(\mathrm{P}<0.001)$. All 10 ablation zones with positive target sign on T2WI exhibited positive target sign on both in- and opposed-phase T1WI. All AMs appeared as areas of high and low SI on postprocedural T1- and T2WI, respectively. However, the SI of the index tumors was variable. When the target sign was correlated with the SI of HCC on pre-procedural MRI, positive target sign was noted in 33 (94.3\%) of 35 HCCs and 30 (96.8\%) of 31 HCCs with low SI on pre-treatment in-phase and opposed-phase imaging, respectively (Table 3). However, positive target sign was only noted in five (50\%) of 10 cases and eight (57.1\%) of 14 cases with iso- or high SI on pretreatment in-phase and opposed-phase T1WI, respectively (Table 3).

\subsection{Comparison of the AM Status Between MDCT and MRI}

Among the 36 RFA zones that showed positive target sign on MDCT, the AM status was classified as AM-plus (n 

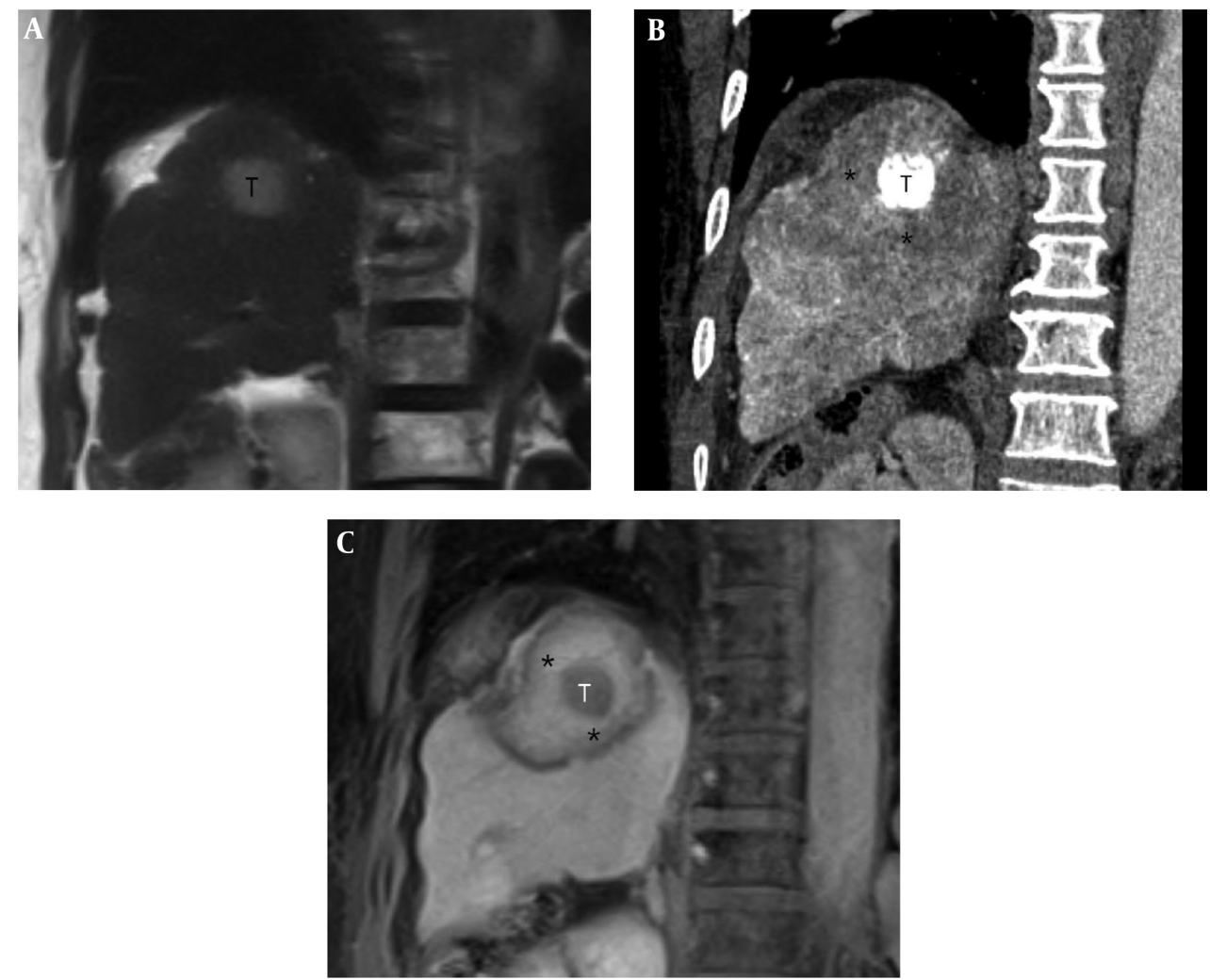

Figure 2. A 62-year-old woman with a 2.6-cm hepatocellular carcinoma (HCC) in the superior segment of the right hepatic lobe. A, Pre-procedural coronal T2-weighted MR image shows a 2.6-cm HCC (T) in the right hepatic dome. B, Post-procedural coronal reformatted CT image demonstrates an index tumor (T) with compact lipiodol uptake in the right hepatic dome that is visually separated from the ablative margin (AM) $\left({ }^{*}\right)$ within the ablation zone. The AM status of was classified as AM-plus. C, Post-procedural coronal contrast-enhanced T1-weighted MR image reveals the index tumor (T) within the ablation zone, which is evident as a low signal intensity (SI) lesion. The AM $\left({ }^{*}\right)$ is visible as an area with high SI and is visually separated from the index tumor. The AM status was classified as AM-plus.

\begin{tabular}{|c|c|c|c|c|c|c|}
\hline \multirow{3}{*}{$\begin{array}{l}\text { Signal intensity of } \\
\text { HCC in } \\
\text { pre-procedural } \\
\text { MRI }\end{array}$} & \multicolumn{6}{|c|}{ Target sign } \\
\hline & \multicolumn{2}{|c|}{ In-phase T1WI } & \multicolumn{2}{|c|}{ Opposed-phase T1WI } & \multicolumn{2}{|c|}{ T2WI } \\
\hline & $+(\mathbf{n}=38)$ & $-(n=7)$ & $+(\mathbf{n}=\mathbf{3 8})$ & $-(\mathbf{n}=7)$ & $+(\mathbf{n}=\mathbf{1 0})$ & $-(\mathbf{n}=35)$ \\
\hline Low SI & 33 & 2 & 30 & 1 & 0 & 2 \\
\hline Isointense & 3 & 3 & 5 & 4 & 1 & 4 \\
\hline High SI & 2 & 2 & 3 & 2 & 9 & 29 \\
\hline
\end{tabular}

Abbreviations: HCC, hepatocellular carcinoma; MRI, magnetic resonance imaging; SI, signal intensity; T1WI, T1 weighted image; T2WI, T2 weighted image

$=27,75 \%)$ and AM-zero $(n=9,25 \%)$, whereas the 40 ablation zones in which the AM status could be evaluated on MRI scans were categorized as AM-plus $(\mathrm{n}=31,77.5 \%)$ and AMzero $(n=9,22.5 \%)$. The ablation zones with negative target sign were categorized as indeterminate with respect the AM status (Table 4). Of 32 RFA zones that showed positive target sign at both MDCT and MRI, 23 (71.9\%) and five (15.6\%) were identically categorized as AM-plus and AM-zero, respectively, on both CT and MR images (strong agreement; $\mathrm{k}=0.634,95 \%$ confidence interval $=0.307-0.961)$

The incidence of LTP was closely correlated with the CTand MR-based classification of AM status on multivariate analysis. LTP occurred in $3.7 \%$ and $3.2 \%$ of the RFA zones with AM-plus status on CT and MRI, respectively. However, among nine cases with AM-zero status on CT and MRI, LTP was observed in four (44.4\%) ablation zones during the follow-up period. 

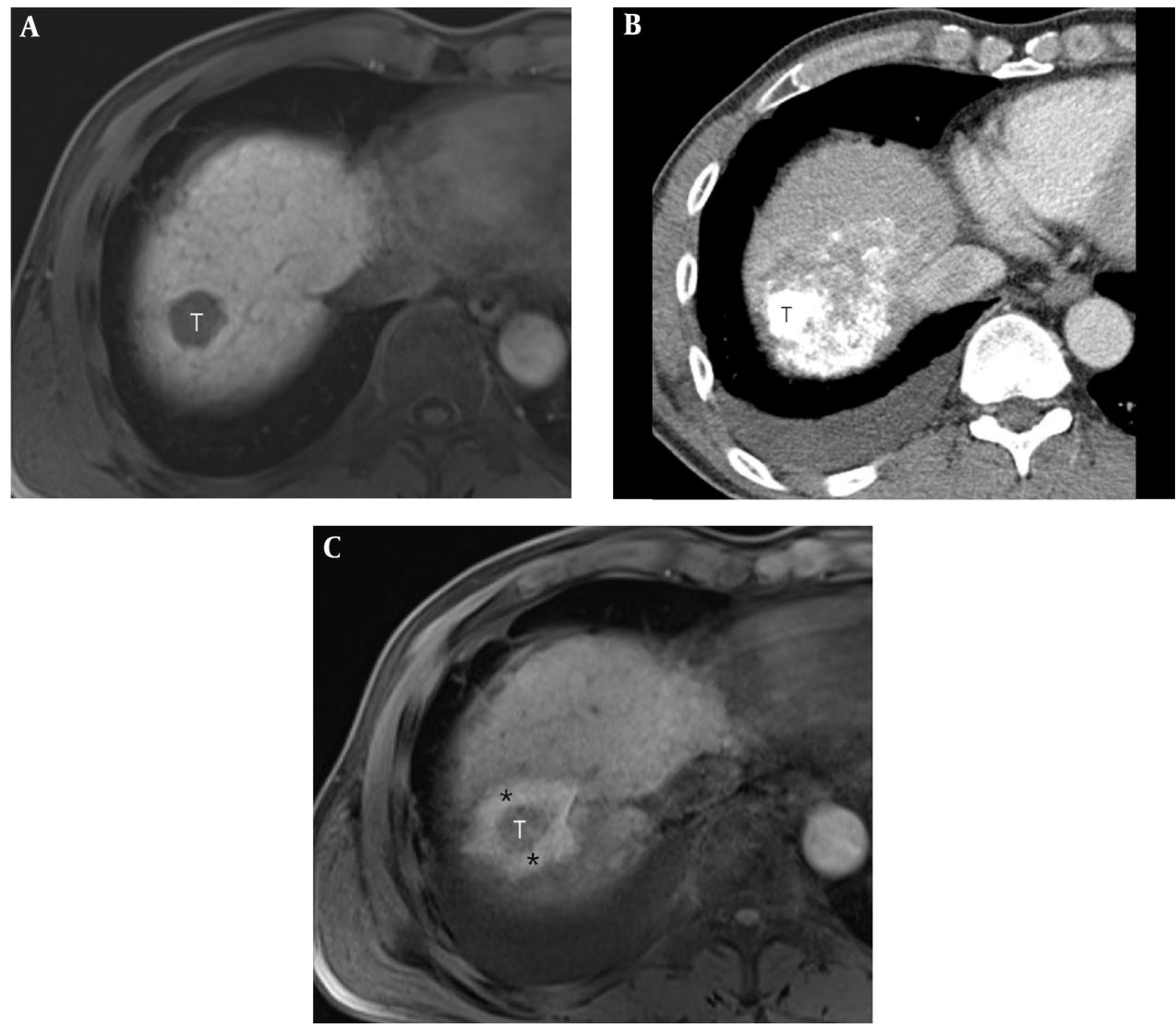

Figure 3. A 49-year-old man with a 2.4-cm hepatocellular carcinoma (HCC) in the right hepatic dome. A, Pre-procedural axial T1-weighted MR image shows a 2.4-cm HCC (T) in the right hepatic dome. B, Post-procedural axial CT image shows the index tumor (T) with compact lipiodol uptake in the right hepatic dome. It is difficult to visually discriminate the index tumor from the ablative margin (AM) due to amorphic and dense deposition of hyper-attenuating iodized oil around the index tumor. C, Post-procedural axial in-phase T1-weighted MR image reveals the index tumor (T) within the ablation zone, which is visible as a low signal intensity (SI)lesion. The AM (*) is visible as an area with high SI and is visually separated from the index tumor. The AM status was classified as AM-plus.

\begin{tabular}{|c|c|c|c|}
\hline & \multicolumn{3}{|c|}{ MRI } \\
\hline & AM plus $(n=31)$ & $\operatorname{AM}$ zero $(\mathbf{n}=9)$ & Indeterminate $^{a}(n=5)$ \\
\hline \multicolumn{4}{|l|}{ MDCT } \\
\hline AM plus $(\mathrm{n}=27)$ & 23 & 2 & 2 \\
\hline${ }^{*}$ Indeterminate $(\mathrm{n}=9)$ & 6 & 2 & 1 \\
\hline
\end{tabular}

Abbreviations: AM, ablative margin; MDCT, multiple detector computed tomography; MRI, magnetic resonance imaging

${ }^{a}$ Indeterminate refers to the cases with negative target sign.

\section{Discussion}

As in RFA alone, immediately after completion of combined therapy with TACE and RFA, imaging evaluation should be performed to determine the need for additional treatment. Evaluation usually involves assessment of the extent of AM surrounding the index tumor within the RFA zone on CT images. However, it is not always straightforward to determine whether the extent of AM is sufficient or not on the basis of visual comparison of pre- and postRFA axial CT images. Instead, several alternative strategies have been proposed to overcome the possibility of inherently inaccurate, axial CT-based evaluation of the technical success of RFA $(9,12,18,33)$. These techniques are aimed at 

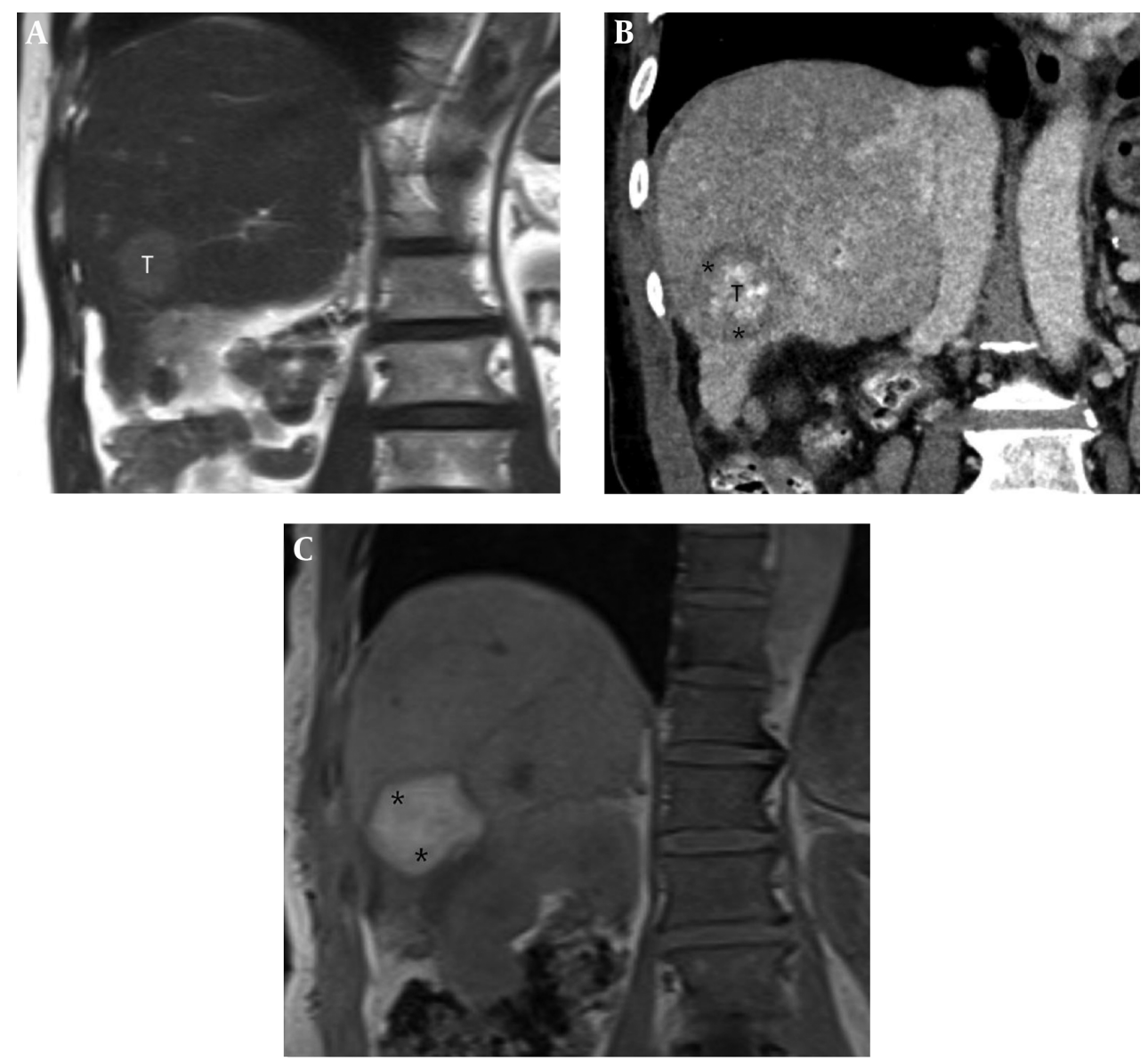

Figure 4. A 71-year-old man with a 2.7-cm hepatocellular carcinoma (HCC) in the inferior segment of the right hepatic lobe. A, Pre-procedural coronal T2-weighted MR image shows a 2.7-cm HCC (T) in the inferior segment of the right hepatic lobe. B, Post-procedural coronal reformatted CT image shows the index tumor (T) with defective lipiodol uptake in the right hepatic lobe, which is visually separated from the ablative margin (AM) $\left({ }^{*}\right)$ within the ablation zone. The AM status was classified as AM-plus. C, Postprocedural coronal in-phase T1-weighted MR image shows an ablation zone with high signal intensity (SI) (*). However, the index tumor is not perceptible within the ablation zone.

pinpointing the exact location of an index tumor within the ablation zone by image fusion of pre- and post-RFA imaging data, thereby enabling an intuitive interpretation of the relationship between the AM and index tumor in a single fused image. However, these image fusion techniques have several drawbacks, such as registration error, prolonged processing time, and poor image quality $(18,33)$.

In addition to several issues related to post-procedural imaging studies in cases with RFA alone, effect of iodized oil should be considered when evaluating treatment success in cases with combined therapy. When infused via the feeding artery in the liver, lipiodol is preferentially distributed in the index tumor and persists for more than several months owing to a siphoning effect originating from tumor neovascularization and the absence of phagocytosis by Kupffer cells within the tumor (34). Further, lipi- odol delivered into the surrounding normal hepatic tissue is stacked up in the portal venules and is cleared within a week (34). Therefore, when iodized oil is used as an embolizing agent, CT-based evaluation is not usually recommended within a month post-procedure because of considerable artifacts generated from lipiodol accumulation in the hepatic parenchyma surrounding the index tumor (20). However, in CT examination of cases with lipiodol retention exclusively in the index tumor, precise localization of the tumor border is possible based on the pattern of lipiodol accumulation (35). Previous studies showed that CT performed at 1-week post-arterial infusion of lipiodol was effective to precisely localize index tumors within the ablation zone $(23,36)$. Thus, CT images with iodized oil marking on index tumors can be used as reference images to assess the ability of MRI to provide visual discrimination between 
the index tumor and $\mathrm{AM}(23)$.

Meanwhile, in CT images obtained immediately after TACE, the accentuated visibility of the index tumor is compromised because of distribution of lipiodol in both the tumors and the surrounding liver. Moreover, the pattern of accumulation of iodized oil in and around the index tumor changes (disrupted or scattered) in patients undergoing RFA after TACE due to elevated pressure within the ablation zone (37). Thus, the pattern of lipiodol accumulation within the index tumor and adjacent liver may be more unpredictable immediately after combined therapy. In our study, visual discrimination between the index tumor and AM was difficult in $20 \%$ of cases due to amorphic and dense accumulation of lipiodol around the index tumor or insufficient lipiodol deposition within the tumor.

During the last decade, several reports have indicated the useful role of MRI in evaluation of the technical success of RFA immediately after the procedure $(17,21-25,38)$. The authors noted that the index tumor was visualized within the ablation zone on pre-contrast and ferucarbotran- or gadoxetic acid-enhanced T1WI. According to previous studies, the target sign was visually identified on the MRIs of $64 \%$ to $96.4 \%$ cases $(22,24,39)$. Because iodized oil does not significantly affect the SI of the HCC and surrounding liver and MRI is not subject to artifacts generated by highly accumulated lipiodol $(20,23)$, the usefulness of MRI in evaluating technical success could be further emphasized in cases of combined therapy with TACE and RFA. Although lipiodol accumulation does not influence the SI on T2WI, De Santis et al. (26) reported that lipiodol retention shortened the T1-relaxation within two to three months after TACE, which may lead to a slight increase in SI at T1WI. However, this effect may be cancelled out when evaluating the presence of target sign within the ablation zone immediately after the procedure because the SI of the ablation zone has tendency to increase with time at T1WI (39).

In our study, MRI showed a positive target sign in $88.9 \%$ of lesions, which is comparable to results reported in previous studies evaluating visual separation of the AM and index tumor on the same image after RFA alone $(22,24,38)$. An earlier study in which technical success was evaluated using MRI showed that the proportion of cases with positive target sign was similar between the two groups (75\% in RFA alone and $81.2 \%$ in combined therapy with TACE and RFA) (17). Thus, it is likely that iodized oil retention would not affect the results of the evaluation of treatment efficacy with MRI.

According to a previous study by Koda et al. (22), isointense tumors on pre-RFA T1WI showed a trend of imperceptibility within the ablation zone. In our study, isointense tumors on pre-procedural MRI showed a positive target sign only in $50 \%, 55.5 \%$, and $20 \%$ of in-phase T1WI, opposed- phase T1WI, and T2WI, respectively. In addition, in our study, a positive target sign was observed less frequently in index tumors that showed high and low SI at pre-RFA T1 and T2WI, respectively. Thus, in this situation, technical success needs to be determined according to the presence or absence of a viable tumor portion around the RFA zone on contrast-enhanced T1WI.

As opposed to an assumption that MDCT would be inferior to MRI for evaluating treatment efficacy due to lipiodol-induced artifacts, our findings indicated absence of significant difference between MDCT and MRI in visual discrimination between the AM and index tumor within the ablation zones immediately after combined treatment with TACE and RFA. This result may be partly explained by the relatively small number of cases (15.5\%) wherein exact localization of lipiodol-retaining tumors within the RFA zone was not possible due to hindrance of irregular distribution of lipiodol around the index tumors.

Our results suggested that imaging evaluation immediately after combined therapy should be performed as follows: First, contrast-enhanced CT is performed to evaluate AM and treatment efficacy; in cases with difficult visual separation of the index tumor with lipiodol marking within the ablation zone, unenhanced T1WI is acquired; and in cases with non-detection of index tumor within the RFA zone on T1WI, technical success should be assessed using contrast-enhanced T1WI.

This study has several limitations. First, histological confirmation of the AM and index tumors was not obtained in all patients. Second, overall survival rate was not compared according to AM status. However, in view of the study's purpose, this may not be a significant drawback in terms of the results obtained. Third, diffusion-weighted MR imaging and subtraction technique between pre- and post-contrast enhanced MR images were not used for evaluating the RFA zones. Fourth, we did not evaluate progressive changes in the pattern of lipiodol accumulation and the SI of the index tumor and AM within the ablation zone at increasing post-procedure time points. Therefore, the results of this study might not be directly applicable to imaging findings at $>$ 7-hour after combined therapy with TACE and RFA.

In conclusion, MDCT and MRI had equivalent ability to differentiate between the AM and index tumor within the ablation zones immediately after RFA combined with TACE. Thus, the two imaging modalities may be complementary assessment tools of treatment efficacy after the procedure. 


\section{Footnotes}

Authors' Contributions: Sang Soo Shin developed the original idea and the protocol, abstracted and analyzed data, wrote the manuscript, and is the guarantor. Sung Mo Kim wrote the manuscript. Byung Chan Lee, Jin Woong Kim, Suk Hee Heo, Chung Hwan Jun, and Yong Yeon Jeong contributed to the development of the protocol, abstracted data, and prepared the manuscript.

Conflict of Interests: None of the authors have any conflict of interests related to the manuscript.

Financial Disclosure: None of the authors have any financial interests related to the material in the manuscript.

Funding/Support: This work was supported by the funds from the National Research Foundation of Korea (2018R1D1A3B07043473), the Chonnam National University Hospital Research Institute of Clinical Medicine in South Korea (CRI18091-2), and DONGKOOK PHARMACEUTICAL CO., LTD. (HCRE13057-7).

\section{References}

1. Kim YS, Lim HK, Rhim H, Lee MW, Choi D, Lee WJ, et al. Ten-year outcomes of percutaneous radiofrequency ablation as first-line therapy of early hepatocellular carcinoma: Analysis of prognostic factors. J Hepatol. 2013;58(1):89-97. doi: 10.1016/j.jhep.2012.09.020. [PubMed: 23023009].

2. Shiina S, Tateishi R, Arano T, Uchino K, Enooku K, Nakagawa H, et al. Radiofrequency ablation for hepatocellular carcinoma: 10-year outcome and prognostic factors. Am J Gastroenterol. 2012;107(4):569-77. quiz 578. doi: 10.1038/ajg.2011.425. [PubMed: 22158026]. [PubMed Central: PMC3321437].

3. Giannini EG, Farinati F, Del Poggio P, Rapaccini GL, Di Nolfo MA, Benvegnu $\mathrm{L}$, et al. Ten-year outcome of radiofrequency thermal ablation for hepatocellular carcinoma: An Italian experience. Am J Gastroenterol. 2012;107(10):1588-9. author reply 1590. doi: 10.1038/ajg.2012.250. [PubMed: 23034617].

4. Forner A, Llovet JM, Bruix J. Hepatocellular carcinoma. Lancet. 2012;379(9822):1245-55. doi: 10.1016/S0140-6736(11)61347-0. [PubMed: 22353262].

5. Livraghi T, Meloni F, Di Stasi M, Rolle E, Solbiati L, Tinelli C, et al. Sustained complete response and complications rates after radiofrequency ablation of very early hepatocellular carcinoma in cirrhosis: Is resection still the treatment of choice? Hepatology. 2008;47(1):82-9. doi: 10.1002/hep.21933. [PubMed: 18008357]

6. Kim JW, Shin SS, Heo SH, Hong JH, Lim HS, Seon HJ, et al. Ultrasoundguided percutaneous radiofrequency ablation of liver tumors: How we do it safely and completely. Korean J Radiol. 2015;16(6):1226-39. doi: 10.3348/kjr.2015.16.6.1226. [PubMed: 26576111]. [PubMed Central: PMC4644743].

7. Peng ZW, Zhang YJ, Chen MS, Liang HH, Li JQ, Zhang YQ, et al Risk factors of survival after percutaneous radiofrequency ablation of hepatocellular carcinoma. Surg Oncol. 2008;17(1):23-31. doi: 10.1016/j.suronc.2007.08.002. [PubMed: 17869095].

8. Kei SK, Rhim H, Choi D, Lee WJ, Lim HK, Kim YS. Local tumor progression after radiofrequency ablation of liver tumors: Analysis of morphologic pattern and site of recurrence. AJR Am J Roentgenol. 2008;190(6):1544-51. doi: 10.2214/AJR.07.2798. [PubMed:18492905].
9. Kim YS, Lee WJ, Rhim H, Lim HK, Choi D, Lee JY. The minimal ablative margin of radiofrequency ablation of hepatocellular carcinoma $(>2$ and $<5 \mathrm{~cm}$ ) needed to prevent local tumor progression: 3D quantitative assessment using CT image fusion. AJR Am J Roentgenol. 2010;195(3):758-65. doi: 10.2214/AJR.09.2954. [PubMed: 20729457].

10. Nakazawa T, Kokubu S, Shibuya A, Ono K, Watanabe M, Hidaka H, et al Radiofrequency ablation of hepatocellular carcinoma: Correlation between local tumor progression after ablation and ablative margin. AJR Am J Roentgenol. 2007;188(2):480-8. doi: 10.2214/AJR.05.2079. [PubMed: 17242258].

11. Kim YS, Rhim H, Cho OK, Koh BH, Kim Y. Intrahepatic recurrence after percutaneous radiofrequency ablation of hepatocellular carcinoma: Analysis of the pattern and risk factors. Eur JRadiol.2006;59(3):432-41. doi:10.1016/j.ejrad.2006.03.007. [PubMed:16690240].

12. Zhong-Zhen S, Kai L, Rong-Oin Z, Er-Jiao X, Ting Z, Ao-Hua Z, et al. A feasibility study for determining ablative margin with 3D-CEUS-CT/MR image fusion after radiofrequency ablation of hepatocellular carcinoma. Ultraschall Med. 2012;33(7):E250-5. doi: 10.1055/s-0032-1325466. [PubMed: 23238803].

13. Lee HJ, Kim JW, Hur YH, Shin SS, Heo SH, Cho SB, et al. Combined therapy of transcatheter arterial chemoembolization and radiofrequency ablation versus surgical resection for single $2-3 \mathrm{~cm}$ hepatocellular carcinoma: A propensity-score matching analysis. J Vasc Intery Radiol.2017;28(9):1240-1247 e3.doi:10.1016/j.jvir.2017.05.015. [PubMed: 28688816]

14. Kim JW, Kim JH, Won HJ, Shin YM, Yoon HK, Sung KB, et al. Hepatocellular carcinomas 2-3 cm in diameter: Transarterial chemoembolization plus radiofrequency ablation vs. radiofrequency ablation alone. Eur J Radiol. 2012;81(3):e189-93. doi: 10.1016/j.ejrad.2011.01.122. [PubMed: 21353417].

15. Kim JH, Won HJ, Shin YM, Kim SH, Yoon HK, Sung KB, et al. Medium sized $(3.1-5.0 \mathrm{~cm})$ hepatocellular carcinoma: transarterial chemoembolization plus radiofrequency ablation versus radiofrequency ablation alone. Ann Surg Oncol. 2011;18(6):1624-9. doi: 10.1245/s10434-0111673-8. [PubMed: 21445671].

16. Yamanaka T, Yamakado K, Takaki H, Nakatsuka A, Shiraki K, Hasegawa $\mathrm{H}$, et al. Ablative zone size created by radiofrequency ablation with and without chemoembolization in small hepatocellular carcinomas. Jpn J Radiol. 2012;30(7):553-9. doi: 10.1007/s11604-012-0087-2. [PubMed: 22610876].

17. Koda M, Tokunaga S, Okamoto T, Hodozuka M, Miyoshi K, Kishina $\mathrm{M}$, et al. Clinical usefulness of the ablative margin assessed by magnetic resonance imaging with Gd-EOB-DTPA for radiofrequency ablation of hepatocellular carcinoma. J Hepatol. 2015;63(6):1360-7. doi: 10.1016/j.jhep.2015.07.023. [PubMed: 26232269].

18. Kim KW, Lee JM, Klotz E, Kim SJ, Kim SH, Kim JY, et al. Safety margin as sessment after radiofrequency ablation of the liver using registration of preprocedure and postprocedure CT images. AJR Am J Roentgenol. 2011;196(5):W565-72. doi: 10.2214/AJR.10.5122. [PubMed: 21512046].

19. Bouda D, Lagadec M, Alba CG, Barrau V, Dioguardi Burgio M, Moussa $\mathrm{N}$, et al. Imaging review of hepatocellular carcinoma after thermal ablation: The good, the bad, and the ugly. J Magn Reson Imaging. 2016;44(5):1070-90. doi:10.1002/jmri.25369. [PubMed: 27505619].

20. Kloeckner R, Otto G, Biesterfeld S, Oberholzer K, Dueber C, Pitton MB MDCT versus MRI assessment of tumor response after transarterial chemoembolization for the treatment of hepatocellular carcinoma. Cardiovasc Intervent Radiol. 2010;33(3):532-40. doi: 10.1007/s00270009-9728-y. [PubMed: 19847482].

21. Koda M, Tokunaga S, Miyoshi K, Kishina M, Fujise Y, Kato J, et al. Ablative margin states by magnetic resonance imaging with ferucarbotran in radiofrequency ablation for hepatocellular carcinoma can predict local tumor progression. J Gastroenterol. 2013;48(11):1283-92. doi: 10.1007/s00535-012-0747-0. [PubMed: 23338488].

22. Koda M, Tokunaga S, Miyoshi K, Kishina M, Fujise Y, Kato J, et al. Assessment of ablative margin by unenhanced magnetic resonance imaging after radiofrequency ablation for hepatocellular carcinoma. Eur 
J Radiol. 2012;81(10):2730-6. doi: 10.1016/j.ejrad.2011.11.013. [PubMed: 22137612].

23. Koda M, Tokunaga S, Fujise Y, Kato J, Matono T, Sugihara T, et al. Assessment of ablative margin after radiofrequency ablation for hepatocellular carcinoma; comparison between magnetic resonance imaging with ferucarbotran and enhanced CT with iodized oil deposition. Eur J Radiol. 2012;81(7):1400-4. doi: 10.1016/j.ejrad.2011.03.004. [PubMed: 21440397].

24. Yoon JH, Lee EJ, Cha SS, Han SS, Choi SJ, Juhn JR, et al. Comparison of gadoxetic acid-enhanced MR imaging versus four-phase multi-detector row computed tomography in assessing tumor regression after radiofrequency ablation in subjects with hepatocellular carcinomas. J Vasc Interv Radiol. 2010;21(3):348-56. doi: 10.1016/j.jvir.2009.11.014. [PubMed: 20116285].

25. Shao GL, Zheng JP, Guo LW, Chen YT, Zeng H, Yao Z. Evaluation of efficacy of transcatheter arterial chemoembolization combined with computed tomography-guided radiofrequency ablation for hepatocellular carcinoma using magnetic resonance diffusion weighted imaging and computed tomography perfusion imaging: A prospective study. Medicine (Baltimore). 2017;96(3). e5518. doi: 10.1097/MD.0000000000005518. [PubMed: 28099329]. [PubMed Central: PMC5279074].

26. De Santis M, Alborino S, Tartoni PL, Torricelli P, Casolo A, Romagnoli R. Effects of lipiodol retention on MRI signal intensity from hepatocellular carcinoma and surrounding liver treated by chemoembolization. Eur Radiol. 1997;7(1):10-6. [PubMed: 9000387].

27. Mitchell DG, Bruix J, Sherman M, Sirlin CB. LI-RADS (liver imaging reporting and data system): Summary, discussion, and consensus of the LI-RADS management working group and future directions. Hepatology. 2015;61(3):1056-65. doi: 10.1002/hep.27304. [PubMed: 25041904].

28. Yoon JH, Park JW, Lee JM. Noninvasive diagnosis of hepatocellular carcinoma: elaboration on Korean Liver Cancer Study GroupNational Cancer Center Korea practice guidelines compared with other guidelines and remaining issues. Korean J Radiol. 2016;17(1):724. doi: 10.3348/kjr.2016.17.1.7. [PubMed: 26798212]. [PubMed Central: PMC4720815].

29. Choi JY, Lee JM, Sirlin CB. CT and MR imaging diagnosis and staging of hepatocellular carcinoma: Part I. Development, growth, and spread: Key pathologic and imaging aspects. Radiology. 2014;272(3):635-54. doi: 10.1148/radiol.14132361. [PubMed: 25153274]. [PubMed Central: PMC4263631].

30. Choi JY, Lee JM, Sirlin CB. CT and MR imaging diagnosis and staging of hepatocellular carcinoma: Part II. Extracellular agents, hepatobiliary agents, and ancillary imaging features. Radiology. 2014;273(1):30-
50. doi: 10.1148/radiol.14132362. [PubMed: 25247563]. [PubMed Central: PMC4263770].

31. Rhim H, Lim HK, Kim YS, Choi D. Percutaneous radiofrequency ablation with artificial ascites for hepatocellular carcinoma in the hepatic dome: Initial experience. AJR Am J Roentgenol. 2008;190(1):91-8. doi: 10.2214/AJR.07.2384. [PubMed: 18094298].

32. Ahmed M, Solbiati L, Brace CL, Breen DJ, Callstrom MR, Charboneau JW, et al. Image-guided tumor ablation: standardization of terminology and reporting criteria-a 10-year update. Radiology. 2014;273(1):241-60. doi: 10.1148/radiol.14132958. [PubMed: 24927329]. [PubMed Central: PMC4263618].

33. Makino Y, Imai Y, Igura T, Hori M, Fukuda K, Sawai Y, et al. Comparative evaluation of three-dimensional Gd-EOB-DTPA-enhanced MR fusion imaging with CT fusion imaging in the assessment of treatment effect of radiofrequency ablation of hepatocellular carcinoma. Abdom Imaging. 2015;40(1):102-11. doi: 10.1007/s00261-014-0201-2. [PubMed: 25052767].

34. Shin SW. The current practice of transarterial chemoembolization for the treatment of hepatocellular carcinoma. Korean J Radiol. 2009;10(5):425-34. doi: 10.3348/kjr.2009.10.5.425. [PubMed: 19721826]. [PubMed Central: PMC2731859].

35. Minami Y, Nishida N, Kudo M. Therapeutic response assessment of RFA for HCC: Contrast-enhanced US, CT and MRI. World J Gastroenterol. 2014;20(15):4160-6. doi: 10.3748/wjg.v20.115.4160. [PubMed: 24764654]. [PubMed Central: PMC3989952].

36. Nishikawa H, Inuzuka T, Takeda H, Nakajima J, Sakamoto A, Henmi $S$, et al. Percutaneous radiofrequency ablation therapy for hepatocellular carcinoma: A proposed new grading system for the ablative margin and prediction of local tumor progression and its validation. J Gastroenterol. 2011;46(12):1418-26. doi: 10.1007/s00535-011-04524. [PubMed: 21845378].

37. Kawamoto C, Yamauchi A, Baba Y, Kaneko K, Yakabi K. Measurement of intrahepatic pressure during radiofrequency ablation in porcine liver.J Gastroenterol. 2010;45(4):435-42. doi: 10.1007/s00535-009-01561. [PubMed: 19936601].

38. Khankan AA, Murakami T, Onishi H, Matsushita M, Iannaccone R, Aoki $\mathrm{Y}$, et al. Hepatocellular carcinoma treated with radio frequency ablation: An early evaluation with magnetic resonance imaging.J Magn Reson Imaging. 2008;27(3):546-51. doi: 10.1002/jmri.21050. [PubMed: 18183580].

39. Kim YS, Rhim H, Lim HK, Choi D, Lee MW, Park MJ. Coagulation necrosis induced by radiofrequency ablation in the liver: histopathologic and radiologic review of usual to extremely rare changes. Radiographics. 2011;31(2):377-90. doi: 10.1148/rg.312105056. [PubMed: 21415185]. 